\title{
The trajectory characteristics and clinical significance of the left-sided lumbar segmental artery: a prospective cross-sectional radio-anatomical study
}

\author{
Weibo Huang ${ }^{1 \#}$, Ping Zhou ${ }^{2 \#}$, Lin Xie ${ }^{1 \#}$, Zhongxiong Huang ${ }^{2}$, Chaojun Zheng ${ }^{1}$, Xiaosheng Ma $^{1}$, \\ Hongli Wang ${ }^{1 *}$, Jianyuan Jiang ${ }^{1 *}$ \\ ${ }^{1}$ Department of Orthopedics, Huashan Hospital, Fudan University, Shanghai, China; ${ }^{2}$ PET-CT Center, Chenzhou First People’s Hospital, Xiangnan \\ University, Chenzhou, China
}

Contributions: (I) Conception and design: J Jiang, H Wang, W Huang; (II) Administrative support: J Jiang, X Ma; (III) Provision of study materials or patients: P Zhou, Z Huang; (IV) Collection and assembly of data: W Huang, L Xie; (V) Data analysis and interpretation: C Zheng, W Huang; (VI) Manuscript writing: All authors; (VII) Final approval of manuscript: All authors.

"These authors contributed equally to this work and should be considered as co-first authors.

*These authors contributed equally to this work and should be considered as co-corresponding author.

Correspondence to: Jianyuan Jiang, MD; Hongli Wang, MD. Department of Orthopedics, Huashan Hospital, Fudan University, 12 Mid-Wulumuqi Road, Shanghai 200040, China. Email: 13301050222@fudan.edu.cn; wanghongli@huashan.org.cn or wanghongli0212@163.com.

Background: Vascular injury to the lumbar segmental arteries is a devastating complication in minimally invasive lumbar interbody fusion. Previous studies on the anatomy of the lumbar segmental arteries are limited. This prospective cross-sectional study aims to quantitatively describe the brief trajectory of the lumbar segmental arteries on the left side (SegAL) and to discuss its clinical significance.

Methods: One hundred and two asymptomatic volunteers were prospectively enrolled and underwent computed tomography angiography (CTA). Anatomical parameters including the existence rate, relative positions and directions of SegAL were measured. Mann-Whitney $U$ tests were performed, and statistical significance was set at $\mathrm{P}<0.05$.

Results: A total of 404 lumbar SegAL were identified. The SegAL of L1, L2 and L3 were identified in all subjects while the L4 SegAL were absent in 9 of 102 (8.8\%) and the L5 SegAL were absent in 97 of 102 (95.1\%) volunteers. In 25 of 97 (25.8\%) volunteers without the L5 SegAL, the branches of the L4 SegAL ran along the disks. Meanwhile, the branches of L3 intersecting over the intervertebral discs (IVD) were found in 8 of $9(88.9 \%)$ subjects without the L4 SegAL and in 4 of 93 (4.3\%) subjects with L4 SegAL. The branch angles between the L1, L2 SegAL and the aorta were significantly acute $(\mathrm{P}<0.05)$. The L3 SegAL ran approximately vertically with the aorta while the branch angles of the L4 SegAL were significantly blunt $(\mathrm{P}<0.05)$. according to the distances measured, on the anterior vertebral walls, the SegAL of L1 and L2 were significantly closer to the inferior vertebral walls than the SegAL of L3 and L4, while on the posterior vertebral walls, the L3 and L4 SegAL were significantly closer to the inferior walls.

Conclusions: Arterial branches may course over the L3-4 and L4-5 IVD spaces and the branches over the L3-4 disks are more likely to be present when L4 segmental arteries are absent, thus posing potential risks of arterial complications. Because of the SegAL adjacent to the disks, the risk of arterial injury may be higher anteriorly at L1 and L2 and higher posteriorly at L3 and L4. 


\begin{abstract}
Keywords: Lumbar segmental arteries; oblique lateral lumbar interbody fusion (OLIF); lateral lumbar interbody
\end{abstract} fusion (LLIF)

Submitted Aug 31, 2021. Accepted for publication Nov 17, 2021.

doi: 10.21037/qims-21-867

View this article at: https://dx.doi.org/10.21037/qims-21-867

\section{Introduction}

The surgical approach of lumbar interbody fusion evolved as the concept and instrument of minimally invasive surgeries developed rapidly in recent years. Among emerging methods, oblique lumbar interbody fusion (OLIF) and lateral lumbar interbody fusion (LLIF) have been widely performed, as they offer various advantages such as shorter operative time, less blood loss and lower risk of injury on related tissues $(1,2)$.

Nevertheless, complications associated with OLIF/LLIF should not be ignored (3-8). Among the complications reported, vascular injury has been considered to be one of the complications with considerable severity and danger $(3,4,8-10)$. Compared with the aorta, the segmental arteries are more likely to be injured with an incidence of $0.3-5 \%$ $(5,7,10,11)$. Postoperative hematoma formation and potential delayed bleeding would affect the clinical efficacy and even be life-threatening $(8,12)$. For surgeons, lack of knowledge and protection of the vascular distribution increases the risk of complications associated with the arteries.

Lumbar segmental arteries originate from the aorta and mainly distribute on the lateral surface of the vertebrae, forming an extensive arterial network that feeds the nerve root, vertebral body and associated muscles or extradural structures (13). Previous studies have evaluated the anatomical parameters of the lumbar segmental arteries associated with OLIF/LLIF and have demonstrated the potential risk of these procedures. Such studies mainly focused on the segmental arteries on the left side (SegAL) given that the OLIF surgeries are mainly performed through the left approach (patients in the right decubitus position) (14). Takata et al. (15) assessed the vascular intervals of adjacent segmental arteries in patients with scoliosis based on computed tomography angiography (CTA), and found that female patients with a Cobb angle $>14.5^{\circ}$ are at risk. However, the main focus of the study was the medium frontal vertebral plane. Orita et al. (16) researched the arterial distribution related to OLIF and described the vascular distribution adjacent to the front lumbar vertebral walls based on magnetic resonance imaging (MRI). However, one limitation of that study was that the accuracy of MRI concerning vascular structures was not satisfactory. Moreover, the study mainly researched the lumbar SegAL close to the anterior walls. The trajectory of the arteries lying on the left vertebral surface remains to be studied.

To address this gap, for more precise and comprehensive trajectory of the segmental arteries lying on the left vertebral surface, the current study used CTA and quantitatively evaluated the anatomical parameters of the SegAL through multiple planes, aiming to supplement the existing information and provide a frame of reference for surgeons during the preoperative and intraoperative decisions.

We present the following article in accordance with the STROBE reporting checklist (available at https://dx.doi. org/10.21037/qims-21-867).

\section{Methods}

\section{Study population}

In this prospective cross-sectional radioanatomical study, 146 volunteers were recruited and 102 healthy volunteers (51 males and 51 females) were enrolled in the final analysis from November 2019 to April 2020 in Huashan Hospital, Fudan University, Shanghai. The final sample size was calculated based on the pre-experiment results involving the CTA images of 24 subjects. The Eq. [1] below was applied to estimate the required sample size (17):

$$
n=\left(\frac{\mu_{\alpha} \sigma}{\delta}\right)^{2}
$$

In this study, $\alpha$ was defined as $0.05\left(\mu_{\alpha}=1.96\right)$. The value of $\sigma$ refers to the estimated standard deviation and a maximum standard deviation of $5.0 \mathrm{~mm}$ concerning the measured distances was obtained during the pre-experiment. The value of $\delta$ represents the margin of error and the value was set as $1.0 \mathrm{~mm}$ in the current study. According to the equation, a final sample size above 96 would be required. 


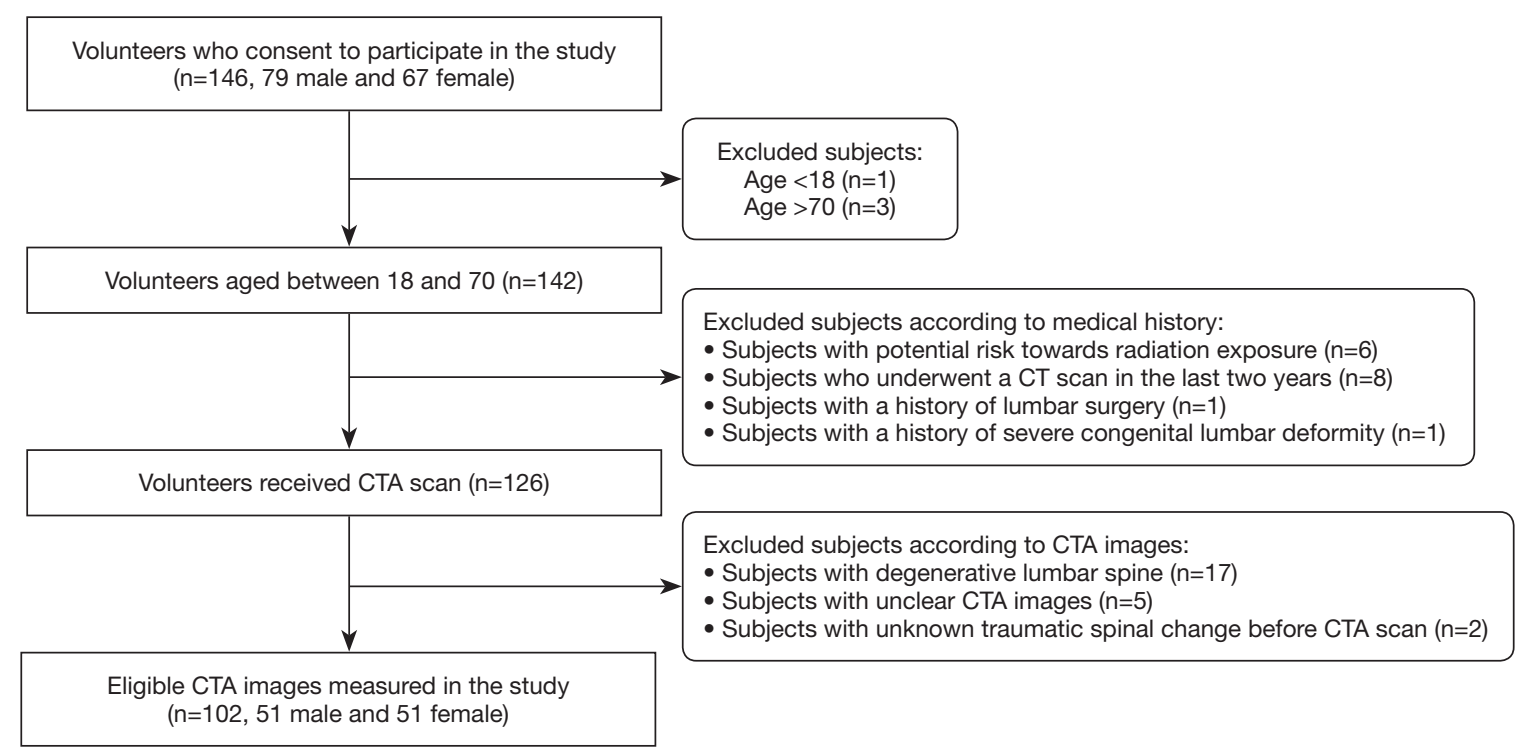

Figure 1 The flow diagram of the participants enrollment process. CTA, computed tomography angiography.

Considering the drop-out or exclusion situations, the number of subjects recruited initially should be more than 120. Meanwhile, based on the sample size and the testing speed during the pre-experiment, a study time span of six months was determined regarding the cost of economical and medical resources.

Figure 1 illustrates the including and excluding procedures during the screening and analysis of this study. The inclusion criterion were asymptomatic adults aged between 18 and 70 who volunteer to participate in the study. Initially, 146 (79 male, 67 female) subjects were recruited for the study between November 2019 to April 2020 and 142 of them were between 18 and 70 . Then subjects with a history of lumbar surgery, congenital lumbar deformity, or other medical history which could possibly disrupt the distribution of the SegAL were excluded. The potential risk caused by radiation exposure was estimated and subjects who had undergone a CT scan in the last two years were excluded either. Thus, 16 subjects were excluded and 126 volunteers remained at this stage and received CTA scan. Subsequently, 19 subjects with radiological presentation of spinal trauma or lumbar spine degeneration were excluded to avoid the bias caused by potential confounders and another 5 subjects were excluded because of unclear images. At last, the CTA images of 102 subjects (51 male, 51 female) were included in the final analysis. The study was conducted in accordance with the Declaration of Helsinki (as revised in 2013) (18). This study was approved by the institutional review board of our hospital (KY-2020-052). All volunteers involved have signed an informed consent letter.

\section{CTA scan and image processing}

CTA was performed with a 64-slice spiral CT scanner (Ingenuity TF CT, Philips, Amsterdam, Netherlands) with a tube voltage of $120 \mathrm{kV}$, a current of $250 \mathrm{~mA}$ and an acquisition matrix of $512 \times 512$ in Huashan Hospital. The tube rotation speed was 1.33 revolution per seconds. The volunteers were placed in a supine position. Lead aprons were used to protect volunteers during the scans. The slice thickness was $0.8 \mathrm{~mm}$, with a slice gap was $0.8 \mathrm{~mm}$. The dose of the contrast was calculated with the protocol of $1.5 \mathrm{~mL} / \mathrm{kg}$. The contrast agent $(35 \mathrm{~g} / 100 \mathrm{~mL}$ Iohexol and $30 \mathrm{~mL} 0.9 \%$ normal saline) were injected by a highpressure injector at a speed of $4.5-5.5 \mathrm{~mL} / \mathrm{s}$. The trigger value was set to $150 \mathrm{HU}$. Images were then processed with Philip Intellispace Portal by applying multiple planar reconstruction and maximum intensity projection when the scans were completed.

\section{Measurement and evaluation}

As shown in Figure 2A, four planes were defined as the anterior, anterior one-fourth, middle, posterior vertebral planes of the corresponding vertebrae. As Figure $2 B$ presents, for each vertebral body, the angles of the 

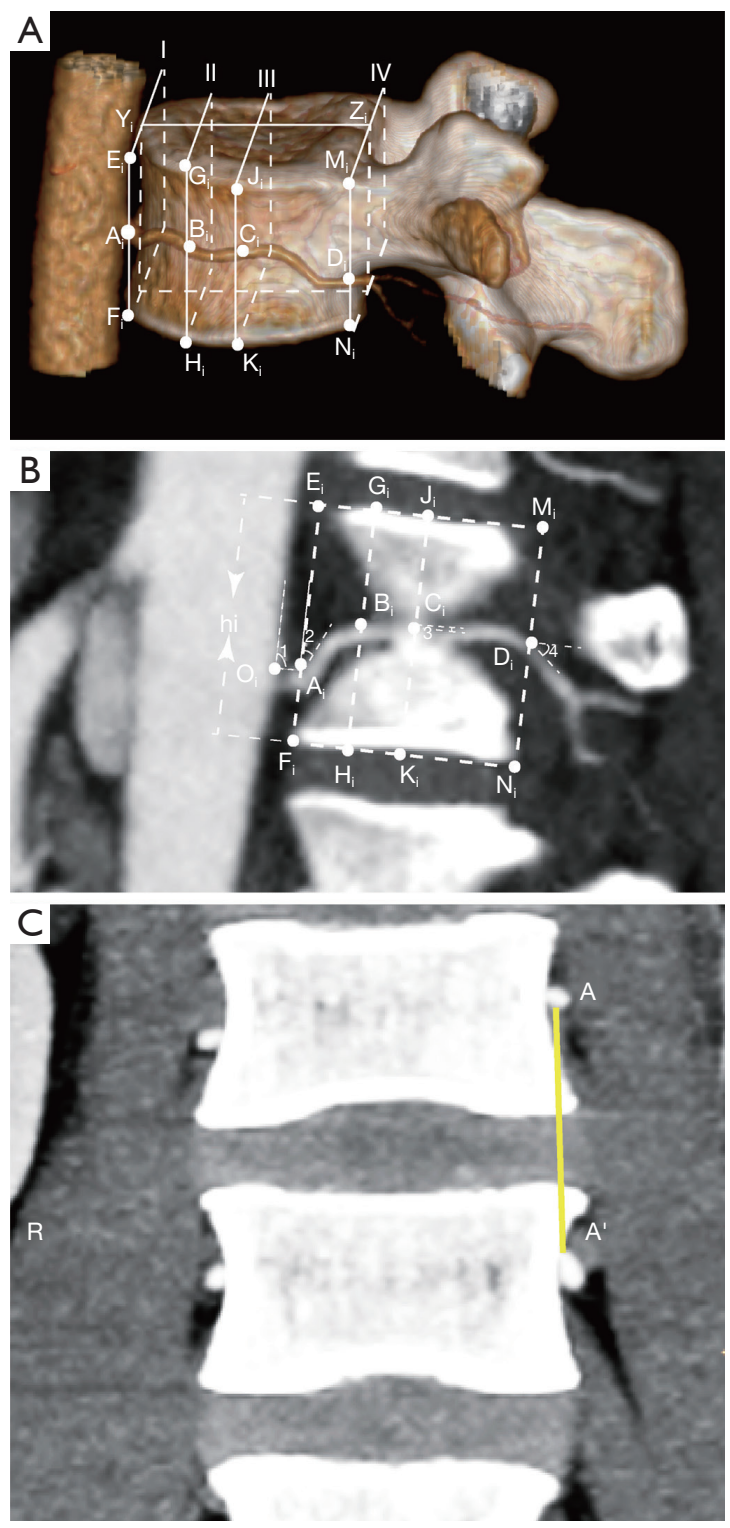

Figure 2 Measurement methods on radiologic images. (A) For each vertebra 'Li', the planes passing through the anterior/posterior borders as well as perpendicular to the sagittal plane were defined as the anterior/posterior vertebral plane (planes I and IV). The paralleled planes passing the anterior one-fourth point and the midpoint of the sagittal axis of the vertebra (YiZi) were defined as the anterior one-fourth and the middle vertebral plane (planes II and III), respectively. The vertebral heights (hi) were measured for analysis. (B) The Oi point is at the start of the SegAL and $\mathrm{Ai}, \mathrm{Bi}, \mathrm{Ci}$ and $\mathrm{Di}$ are the points where the SegAL pass the anterior, one-fourth, middle, posterior planes of vertebrae. $\angle 1$ and $\angle 2$ are angles between the arteries and the longitudinal axis of the aorta at Oi and Ai points. $\angle 3$ and $\angle 4$ are angles between the arteries and the inferior vertebral walls at the $\mathrm{Ci}$ and Di points. $\angle 3$ and $\angle 4$ with a direction towards the inferior vertebral walls were recorded as negative numbers. Distances between the arteries and the superior/inferior walls were measured. If the AiFi, BiHi, CiKi and DiNi were below the inferior vertebral border or the $\mathrm{AiEi}, \mathrm{BiGi}, \mathrm{CiJi}$, DiMi were above the superior vertebral border, the distances were recorded as negative numbers. (C) For each $\mathrm{Li}$, the craniocaudal intervals AiA'i, BiB'i and CC'i were measured on the coronal slices at Ai, Bi and Ci points respectively. SegAL, segmental arteries on the left side. 
Table 1 Demographics of asymptomatic volunteers

\begin{tabular}{|c|c|}
\hline Variables & $\begin{array}{c}\text { Number of subjects } \\
(\%)\end{array}$ \\
\hline \multicolumn{2}{|l|}{ Gender } \\
\hline Male & $51(50.0)$ \\
\hline Female & $51(50.0)$ \\
\hline Ethnicity (Chinese) & $102(100.0)$ \\
\hline \multicolumn{2}{|c|}{ Age (mean \pm SD: $48.2 \pm 7.9$ years old, range, $18-60$ ) } \\
\hline $18 \leq$ age $<20$ & $1(1.0)$ \\
\hline $20 \leq$ age $<30$ & $6(5.9)$ \\
\hline $30 \leq$ age $<40$ & $12(11.8)$ \\
\hline $40 \leq$ age $<50$ & $38(37.3)$ \\
\hline $50 \leq$ age $<60$ & $44(43.1)$ \\
\hline $60 \leq$ age $<70$ & $1(1.0)$ \\
\hline \multicolumn{2}{|c|}{ BMI (mean \pm SD: $21.8 \pm 2.1 \mathrm{~kg} / \mathrm{m}^{2}$, range, $17.2-26.2$ ) } \\
\hline $\mathrm{BMI}<18.5$ & $6(5.9)$ \\
\hline $18.5 \leq \mathrm{BMI}<23$ & $72(70.6)$ \\
\hline $23 \leq \mathrm{BMI}<25$ & $22(21.6)$ \\
\hline $\mathrm{BMI} \geq 25$ & $2(2.0)$ \\
\hline \multicolumn{2}{|l|}{ Occupation $^{\dagger}$} \\
\hline White-collar workers & $35(34.3)$ \\
\hline Pink-collar workers & $27(26.5)$ \\
\hline Blue-collar workers & $20(19.6)$ \\
\hline Agribusiness and low-level workers & $15(14.7)$ \\
\hline Other (students/unemployed workers) & $5(4.9)$ \\
\hline \multicolumn{2}{|l|}{ Smoking } \\
\hline Yes & 36 (35.3) \\
\hline No & $66(64.7)$ \\
\hline
\end{tabular}

${ }^{\dagger}$, white-collar workers include workers with slight manual work such as managers, officers and professionals; pink-collar workers refer to workers with mild to moderate manual work such as service and sale workers; blue-collar workers include mechanical operators and technicians with moderate-to-heavy manual work; agribusiness and low-level workers refer to agriculture workers and low-level laborers with heavy labor work. BMI, body mass index; SD, standard deviation. corresponding SegAL through the anterior, middle and posterior planes of the vertebrae were measured on sagittal slices. Next, distances between the SegAL and the superior/ inferior borders of the vertebrae were measured on sagittal slices as well. And the relative distances to the inferior walls were calculated with the heights of the corresponding vertebrae to decrease the influence of individual difference caused by potential confounders such as the physique, exercise or labor intensity (relative distances $=$ the measured distances/the heights of the corresponding vertebrae). Intervals between adjacent $\mathrm{SegAL}$ were measured on coronal slices (Figure 2C). All measurements were performed by an orthopedic surgeon and a radiologist who were blinded to the volunteers' information.

\section{Statistical analysis}

Statistical analysis was performed using SPSS Statistics version 21.0 (IBM Corp. Armonk, NY, USA). Continuous variables were described as mean \pm standard deviation $(\mathrm{SD})$. Descriptive statistics were used to summarize the variations in anatomical parameters. The Mann-Whitney tests were applied to compare the distances or angles of the SegAL at different levels. Statistical significance was set at $\mathrm{P}<0.05$.

\section{Results}

\section{The presence of the SegAL}

As shown in Figure 1, 102 volunteers (51 males and 51 females) with an average age of $48.2 \pm 7.9$ years (range, $19-60$ years) and an average BMI of $21.8 \pm 2.1 \mathrm{~kg} / \mathrm{m}^{2}$ (range, $17.2-26.2 \mathrm{~kg} / \mathrm{m}^{2}$ ) were included in the analysis. The detailed demographics of the volunteers are presented in Table 1 . The presence of SegAL of L1, L2 and L3 was confirmed in all subjects, whereas the SegAL of L4 were found in 93 of them $(91.2 \%)$ and the SegAL of L5 were identified only in 5 volunteers (4.9\%). However, as Figure 3 shows, in 25 of 97 (25.8\%) volunteers, the branches of the L4 SegAL ran along the disks. Meanwhile, the branches of L3 intersecting over the intervertebral discs (IVD) were found in 8 of 9 (88.9\%) subjects without L4 SegAL and in 4 of 93 (4.3\%) 


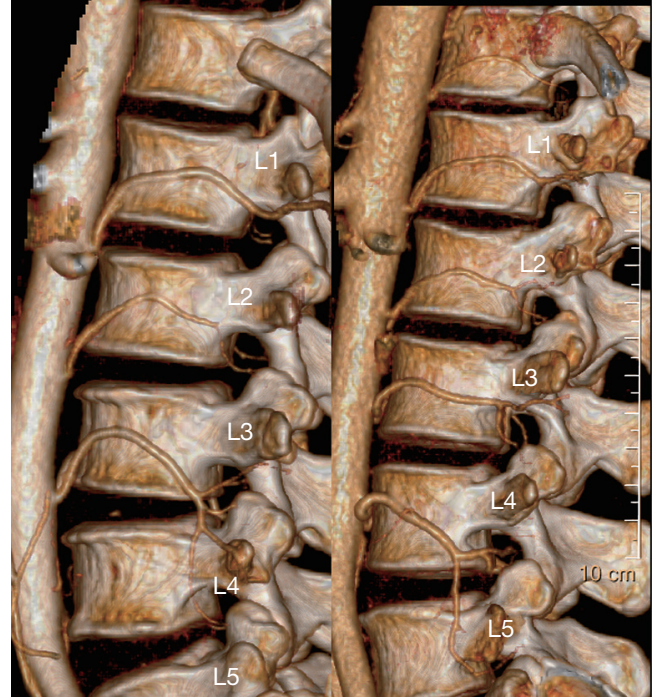

Figure 3 The branches of L3 and L4 SegAL possibly run along the posterolateral aspect of IVD. The branches of the L3 and L4 SegAL could run along the intervertebral disk spaces in subjects without the L4 or L5 SegAL. SegAL, segmental arteries on the left side.

subjects with L4 SegAL.

\section{The directions of the SegAL}

The angles of the SegAL were measured on sagittal images, and the results of which are shown in Table 2. The branch angles between the L1, L2 SegAL and the aorta were significantly acute $(\mathrm{P}<0.05)$. The $\mathrm{L} 3 \mathrm{SegAL}$ ran approximately in an approximately vertical direction with the aorta, while the branch angles of the L4 SegAL were significantly blunt $(\mathrm{P}<0.05)$. On the anterior vertebral walls, the SegAL of L1, L2 and L3 continued to move cranially. In summary, Table 2 indicated that the SegAL of L1, L2 commonly move in a cranial direction while the L4 SegAL generally move in a caudal direction.

\section{The distances between the SegAL and the superior/inferior vertebral walls}

The distances between the SegAL and the inferior/superior borders of the vertebrae were measured on the sagittal slices, and the relative distances to the inferior or superior walls were calculated (relative distances $=$ the measured distances/the heights of the corresponding vertebrae), results of which are presented in Table 3 and Figure 4. On the anterior walls, the relative distances between the SegAL
Table 2 The directions of the SegAL through different planes

\begin{tabular}{|c|c|c|c|c|}
\hline Variables & $\begin{array}{l}\mathrm{O}(\angle 1)^{\dagger},\left(^{\circ}\right) \\
\text { mean } \pm \mathrm{SD}\end{array}$ & $\begin{array}{l}A(\angle 2),\left({ }^{\circ}\right), \\
\text { mean } \pm S D\end{array}$ & $\begin{array}{l}\mathrm{C}(\angle 3),\left({ }^{\circ}\right) \\
\text { mean } \pm \mathrm{SD}\end{array}$ & $\begin{array}{l}\mathrm{D}(\angle 4),\left(^{\circ}\right), \\
\text { mean } \pm \mathrm{SD}\end{array}$ \\
\hline L1 SegAL & $32.9 \pm 38.5$ & $30.1 \pm 17.2^{*}$ & $10.4 \pm 12.4^{*}$ & $-36.8 \pm 23.3$ \\
\hline L2 SegAL & $40.0 \pm 41.1$ & $36.0 \pm 18.4$ & $4.5 \pm 16.4$ & $-40.9 \pm 27.9$ \\
\hline L3 SegAL & $87.4 \pm 45.1^{*}$ & $41.2 \pm 29.0$ & $-11.0 \pm 22.8$ & $-37.7 \pm 24.5$ \\
\hline L4 SegAL & $134.6 \pm 28.6^{\star \star}$ & $77.2 \pm 34.6$ & $-32.3 \pm 22.9^{\star *}$ & $-24.8 \pm 24^{*}$ \\
\hline \multicolumn{5}{|c|}{ 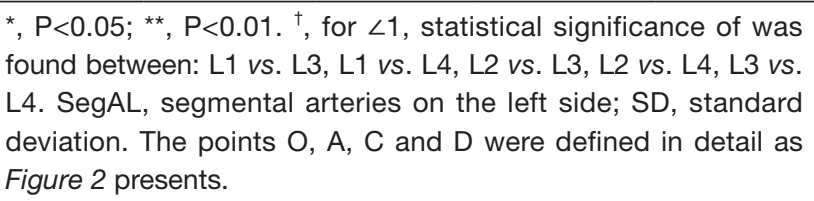 } \\
\hline
\end{tabular}

and the inferior vertebral borders increased gradually from 0.10 in the L1 SegAL to 0.71 in the L4 SegAL. When passing through the anterior one-fourth vertebral planes, the SegAL of L1 and L2 were closer to the inferior border of the relative vertebrae than the arteries of L3 and L4. The SegAL of L1-4 was found to almost reach the central regions of the vertebrae when coursing through the middle vertebral planes. Contrary to the results of the anterior planes, the relative distances between the SegAL and the inferior vertebral borders on the posterior vertebral walls decreased from 0.42 in L1 to 0.16 in L4.

\section{The vascular intervals between adjacent $\operatorname{Seg} A L$}

The coronal slices through the anterior walls, the anterior one-fourth of the vertebrae and the middle vertebrae were captured. On the above three planes, the craniocaudal vascular intervals on the recorded slices are shown in Table 4. The variation trends are shown in Figure 5. Two trends could be found: (I) at the same interval, from the anterior plane to the middle plane, the distances between adjacent SegAL gradually increase; (II) for the same plane, from L1 to L4, the distances between adjacent SegAL gradually increase.

\section{Discussion}

In the current study, the SegAL of L1-3 were identified in all subjects, while the presence of the L5 SegAL was extremely low, with an existence rate of $4.9 \%$. In addition, the L4 SegAL was found in nine subjects. Nevertheless, the L3-4 or L4-5 IVD spaces were not wholly free of vascular structures in subjects without the L4 or L5 SegAL because branches derived from the L3 SegAL were found 
Table 3 The distances and relative distances between the SegAL and inferior/superior vertebral walls (mm) ${ }^{\dagger}$

\begin{tabular}{|c|c|c|c|c|c|c|c|c|}
\hline Variables & $\mathrm{AF}$ & $A E$ & $\mathrm{BH}$ & $B G$ & $\mathrm{CK}$ & CJ & DN & DM \\
\hline L1 SegAL & $\left(0.10 \pm 0.17^{\star \star}\right)$ & $\left(0.92 \pm 0.19^{\star \star}\right)$ & $(0.43 \pm 0.08)$ & $(0.59 \pm 0.10)$ & $(0.51 \pm 0.06)$ & $(0.54 \pm 0.06)$ & $\left(0.42 \pm 0.1^{\star}\right)$ & $(0.68 \pm 0.1)$ \\
\hline \multirow[t]{2}{*}{ L2 SegAL } & $5.0 \pm 4.4$ & $19.5 \pm 4.8$ & $11.0 \pm 2.3$ & $13.5 \pm 2.7$ & $12.1 \pm 1.8$ & $13.1 \pm 1.9$ & $8.7 \pm 2.4$ & $17.0 \pm 2.7$ \\
\hline & $\left(0.21 \pm 0.18^{\star}\right)$ & $\left(0.81 \pm 0.20^{\star}\right)$ & $(0.46 \pm 0.10)$ & $(0.56 \pm 0.11)$ & $(0.50 \pm 0.07)$ & $(0.54 \pm 0.08)$ & $\left(0.36 \pm 0.10^{\star}\right)$ & $(0.71 \pm 0.11)$ \\
\hline L3 SegAL & $\left(0.42 \pm 0.20^{\star}\right)$ & $\left(0.58 \pm 0.20^{\star \star}\right)$ & $\left(0.56 \pm 0.09^{\star}\right)$ & $\left(0.43 \pm 0.09^{\star}\right)$ & $(0.50 \pm 0.07)$ & $(0.51 \pm 0.07)$ & $\left(0.23 \pm 0.12^{*}\right)$ & $\left(0.80 \pm 0.12^{\star}\right)$ \\
\hline \multirow[t]{2}{*}{ L4 SegAL } & $18.0 \pm 5.0$ & $7.5 \pm 4.9$ & $16.3 \pm 2.6$ & $9.0 \pm 2.2$ & $12.5 \pm 2.6$ & $12.8 \pm 2.6$ & $3.6 \pm 2.4$ & $21.8 \pm 3.7$ \\
\hline & $\left(0.71 \pm 0.19^{\star \star}\right)$ & $\left(0.29 \pm 0.19^{\star \star}\right)$ & $\left(0.64 \pm 0.09^{\star}\right)$ & $\left(0.35 \pm 0.08^{\star}\right)$ & $(0.49 \pm 0.10)$ & $(0.50 \pm 0.10)$ & $\left(0.16 \pm 0.10^{\star}\right)$ & $\left(0.85 \pm 0.12^{\star}\right)$ \\
\hline
\end{tabular}

${ }^{\dagger}$, the values were presented in the form of mean \pm standard deviation, the values within the round brackets represents the relative distances (relative distances = measured distances/the corresponding vertebral heights). The distances AF, AE, BH, BG, CK, CJ, DN and DM were described specifically in Figure 2. *, $\mathrm{P}<0.05$; ${ }^{* *}, \mathrm{P}<0.01$. SegAL, segmental arteries on the left side.

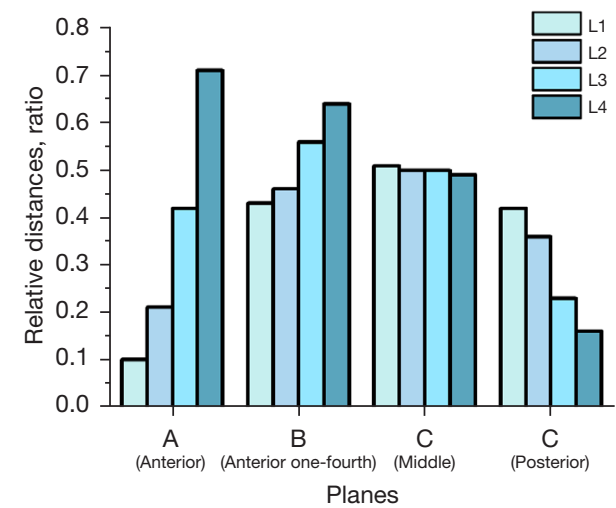

Figure 4 Relative distances between the inferior vertebral borders and the SegAL through different planes. The figure shows the comparison between the distances of different levels and planes. An increasing trend of the distances was found on the anterior vertebral planes from L1 to L4 while a decreasing trend of the distances was found on the posterior planes. The segmental arteries from L1 to L4 were located near the center of the vertebrae. SegAL, segmental arteries on the left side.

Table 4 The Intervals between adjacent SegAL ( $\mathrm{mm})$

\begin{tabular}{lccc}
\hline Variables & AA' $^{\prime}($ mean $\pm \mathrm{SD})$ & $\mathrm{BB}^{\prime}($ mean $\pm \mathrm{SD})$ & $\mathrm{CC}^{\prime}($ mean $\pm \mathrm{SD})$ \\
\hline L1-2 & $27.5 \pm 3.3^{\star *}$ & $31.8 \pm 2.9^{\star}$ & $33.6 \pm 3.2^{\star \star}$ \\
L2-3 & $28.7 \pm 3.2^{*}$ & $33.0 \pm 2.9^{\star}$ & $35.5 \pm 3.0^{\star}$ \\
L3-4 & $30.2 \pm 5.0^{\star *}$ & $35.1 \pm 3.3^{\star *}$ & $36.7 \pm 3.8^{\star}$
\end{tabular}

${ }^{*}, \mathrm{P}<0.05 ;{ }^{*}, \mathrm{P}<0.01$. SegAL, segmental arteries on the left side; $\mathrm{SD}$, standard deviation.

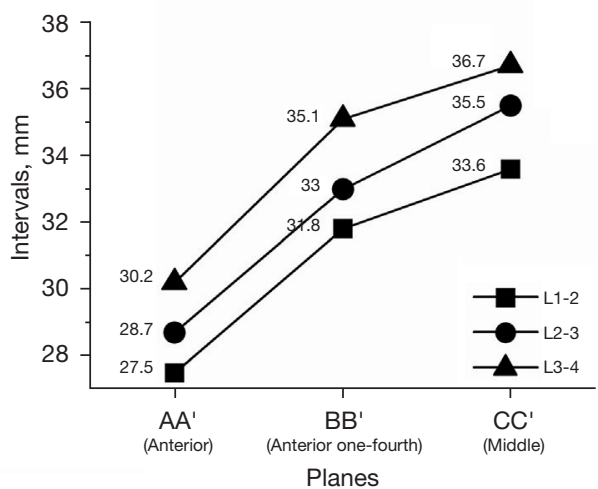

Figure 5 Vascular intervals between the adjacent SegAL through various planes. As the figure shows, for each vertebra, the average intervals on the middle coronal planes were the largest while the average intervals on the anterior coronal planes were the smallest. In addition, for each plane, the average intervals between L3 and L4 were the largest while average the intervals between L1 and L2 were the smallest. SegAL, segmental arteries on the left side.

to course over the L3-4 disks in the eight of nine $(88.9 \%)$ subjects without L4 SegAL, and branches of the L4 SegAL were found to run across the L4-5 disks in 25 of 97 (25.8\%) volunteers without the L5 SegAL (Figure 3). The presence of the branches within the IVD space was more frequent at the L4-5 disks than at the L3-4 disks, which is consistent with a previous study of Nojiri et al. (19). As shown in Figure 3, the current study clearly demonstrated the existence of the branches within the posterolateral L3-4 
A

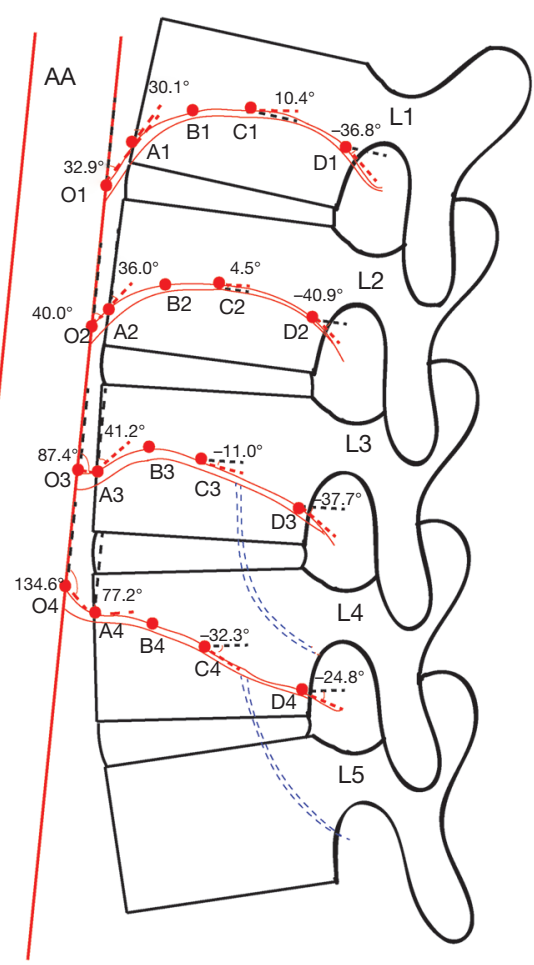

B

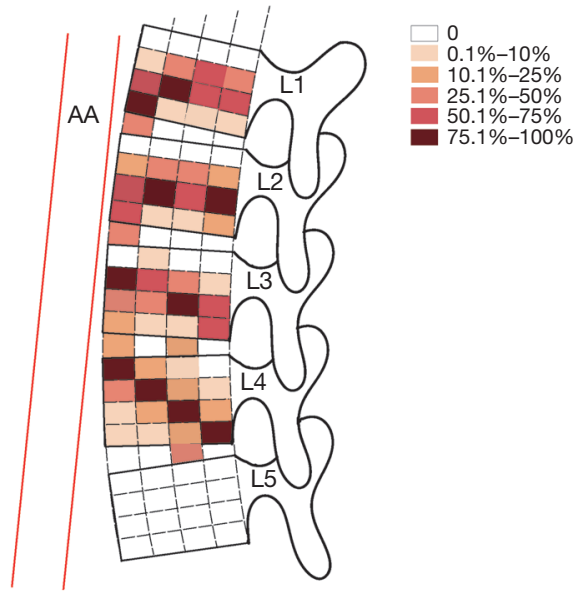

Figure 6 Scheme of the trajectory and distribution frequency of SegAL. (A) Scheme of the trajectory according to the average distances and angles measured in the study. The dotted lines represent the possible presence of arteries within the intervertebral disc spaces. The scheme aims to show the various trajectories between the SegAL of different lumbar levels and to illustrate the general trajectory. (B) Scheme showing the frequency of the vascular distribution. A darker color represents higher frequency and risk of vascular injury. The scheme may help surgeons to protect the SegAL. SegAL, segmental arteries on the left side.
IVD and L4-5 IVD spaces and also discovered the branches of the L3 SegAL coursing over the disks in subjects without the L4 SegAL. Moreover, the existence rate of the branches coursing over the L3-4 disks was found to be higher in subjects without the L4 SegAL than subjects with the L4 SegAL (88.9\% versus $4.3 \%$ ). As for the existence rate of the L5 SegAL, the existence rate of L5 SegAL was obviously lower than that of L1, L2 and L3 SegAL $(15,20,21)$. The absence of the L5 SegAL could be potentially associated with disc degeneration, indicating the significance of protecting the segmental arteries $(20,22)$.

Admittedly, preoperative angiography is not routinely performed for lateral interbody fusion. However, insufficient attention has been paid to the vascular distribution on the lateral surface of the lumbar vertebrae. Limited knowledge concerning the arteries may increase the risk of vascular injury. For instance, the SegAL adjacent to the psoas could be injured during LLIF surgery if surgeons overlook the arteries lying on the lateral vertebrae surface, especially when the surgical field is not clear. According to the current study and previous research, preoperative CTA could be considered to decrease the potential risk of arterial injury in (I) patients with a history of previous surgery or radiotherapy to the abdomen or spine; (II) patients with a history of malignancies; (III) patients with lumbar spinal deformity due to structural damage, inflammation or other reasons; (IV) elderly patients and (V) patients who would undergo OLIF/LLIF surgery at L1-2 or L3-4 segments $(12,15,23)$. Surgeons might balance the radiation exposure and the risk of arterial complications in different individuals. Given that most lateral lumbar fusions are performed without preoperative CTA, the statistically significant anatomical findings of this study could provide information concerning some typical variations and remind surgeons of the potential risks of vascular injury. Figure 6 presents a brief scheme of the trajectory and distribution frequency of SegAL, which could be useful for spine surgeons.

The SegAL should be noted and protected by surgeons during OLIF/LLIF surgery. Specifically, during the pin fixation and exposure procedures, previous reports have advised surgeons to insert needles into the junction of the anterior third with the middle third of IVD space for the OLIF surgery (24). However, the results of this study indicate that surgeons should install the pins and expose the surgical field more flexibly. For instance, according to previous studies, some surgeons recommend to installing 
pins on the inferior half of the vertebrae during the OLIF surgery. However, the current study revealed a lower risk of vascular injury if the surgeons inserted the pins next to the L2 superior endplates when handling the L1-2 IVD space, and next to L3 or L4 inferior endplates when performing OLIF for L3-4 or L4-5 segments. For LLIF surgery, the psoas should be dissected cautiously to protect not only the nerve plexus but also the arteries. Similarly, it is recommended that the pins be installed below the horizontal middle line of L1-2 and L2-3 IVD and above the middle line at L3-4 IVD. Surgeons were recommended that the initial pin should be inserted using the index finger as protection taking care not to leave any structures under valves of the retractor to avoid vascular injuries (24). Additionally, attention should be paid during retractor installation due to possible existence of branches within the L3-4 or L4-5 IVD spaces (Figure 3).

SegAL injury can also occur during disk resection procedures. The SegAL of L1 and L2 could possibly reach the bottom of the vertebrae or even run across the L1-2 or L2-3 disks, and thus caution should be exhibited during the resection of the anterior disks. Compared with the SegAL of L1 and L2, the SegAL of L3 and L4 are at a higher risk of vascular injury during the resection of the posterior part of the L3-4 and L4-5 disks since the arteries possibly left the posterior vertebral walls at comparatively low positions. Meanwhile, the possible existence of the vascular branches running along the disks was noteworthy during the resection of the posterior part of the L3-4 and L4-5 disks, especially in the subjects without the L4 or L5 SegAL. In addition, the risks of vascular injury during the removal procedure of anterior disks possibly exists because the L4 SegAL may run across the L3-4 IVD or located at the top on the front walls of L4. Beckman et al. (13) reported a contralateral psoas hematoma caused by a segmental artery injury during the release of the contralateral annulus. Hence, balancing the risks and surgical efficacy based on preoperative radiologic evaluation and intraoperative checks were essential when applying surgical maneuvers. In addition, the arteries discussed above could also be injured by aggressive endplate preparation and cage insertion. Executing orthogonal maneuvers (forming an orthogonal angle with the instruments positioned perpendicularly to the sagittal plane of the vertebral body) could help resolve this issue (24). The planes researched in this study were all perpendicular to the sagittal plane of the vertebrae, which facilitates the understanding and application of orthogonal maneuvers.
The current study has some limitations. First, all subjects were Chinese; thus, the analysis and results may not be generalizable to other ethnic groups. Second, we only included volunteers who were scanned in the supine position, and hence a possibility of slight translocation of the SegAL when patients were placed in the right decubitus position cannot be discarded. Third, the discussion on the L5 SegAL was insufficient because of the low presence of the L5 SegAL. Finally, the conclusion of the study may not be directly applicable to the management towards patients with disrupted distribution of the SegAL.

\section{Conclusions}

The trajectories and anatomical parameters, such as the relative positions and directions of the segmental arteries, vary between different lumbar levels. The SegAL adjacent to the inferior walls of the L1 and L2 vertebrae or to the superior walls of the L4 vertebrae should be protected during the operation. In addition, attention should be paid during the procedure involving the posterior parts of the L3-4 and L4-5 IVD spaces. Awareness of the need to protect the segmental arteries and proper preoperative radiologic evaluation could help avoid complications.

\section{Acknowledgments}

Funding: This work was supported by grant from The Support Project of Biomedical Technology of Shanghai Science and Technology Innovation Action Plan (No. 19441902200).

\section{Footnote}

Reporting Checklist: The authors have completed the STROBE reporting checklist. Available at https://dx.doi. org/10.21037/qims-21-867

Conflicts of Interest: All authors have completed the ICMJE uniform disclosure form (available at https://dx.doi. org/10.21037/qims-21-867). All authors report that this work was supported by grant from The Support Project of Biomedical Technology of Shanghai Science and Technology Innovation Action Plan (No. 19441902200). The authors have no other conflicts of interest to declare.

Ethical Statement: The authors are accountable for all aspects of the work in ensuring that questions related 
to the accuracy or integrity of any part of the work are appropriately investigated and resolved. The study was conducted in accordance with the Declaration of Helsinki (as revised in 2013). The study was approved by Huashan Hospital of Fudan University (Shanghai, China) and informed consent was taken from all individual participants.

Open Access Statement: This is an Open Access article distributed in accordance with the Creative Commons Attribution-NonCommercial-NoDerivs 4.0 International License (CC BY-NC-ND 4.0), which permits the noncommercial replication and distribution of the article with the strict proviso that no changes or edits are made and the original work is properly cited (including links to both the formal publication through the relevant DOI and the license). See: https://creativecommons.org/licenses/by-nc-nd/4.0/.

\section{References}

1. Gu G, Zhang H, Fan G, He S, Cai X, Shen X, Guan X, Zhou X. Comparison of minimally invasive versus open transforaminal lumbar interbody fusion in two-level degenerative lumbar disease. Int Orthop 2014;38:817-24.

2. Schizas C, Tzinieris N, Tsiridis E, Kosmopoulos V. Minimally invasive versus open transforaminal lumbar interbody fusion: evaluating initial experience. Int Orthop 2009;33:1683-8.

3. Rodgers WB, Gerber EJ, Patterson J. Intraoperative and early postoperative complications in extreme lateral interbody fusion: an analysis of 600 cases. Spine (Phila $\mathrm{Pa}$ 1976) 2011;36:26-32.

4. Harrington JF Jr. Far lateral disc excision at L5-S1 complicated by iliolumbar artery incursion: case report. Neurosurgery 2001;48:1377-9; discussion 1379-80.

5. Fujibayashi S, Kawakami N, Asazuma T, Ito M, Mizutani J, Nagashima H, Nakamura M, Sairyo K, Takemasa R, Iwasaki M. Complications Associated With Lateral Interbody Fusion: Nationwide Survey of 2998 Cases During the First 2 Years of Its Use in Japan. Spine (Phila Pa 1976) 2017;42:1478-84.

6. Jin J, Ryu KS, Hur JW, Seong JH, Kim JS, Cho HJ. Comparative Study of the Difference of Perioperative Complication and Radiologic Results: MIS-DLIF (Minimally Invasive Direct Lateral Lumbar Interbody Fusion) Versus MIS-OLIF (Minimally Invasive Oblique Lateral Lumbar Interbody Fusion). Clin Spine Surg 2018;31:31-6.

7. Sato J, Ohtori S, Orita S, Yamauchi K, Eguchi Y, Ochiai
N, Kuniyoshi K, Aoki Y, Nakamura J, Miyagi M, Suzuki M, Kubota G, Inage K, Sainoh T, Fujimoto K, Shiga Y, Abe K, Kanamoto H, Inoue G, Takahashi K. Radiographic evaluation of indirect decompression of mini-open anterior retroperitoneal lumbar interbody fusion: oblique lateral interbody fusion for degenerated lumbar spondylolisthesis. Eur Spine J 2017;26:671-8.

8. Silvestre C, Mac-Thiong JM, Hilmi R, Roussouly P. Complications and Morbidities of Mini-open Anterior Retroperitoneal Lumbar Interbody Fusion: Oblique Lumbar Interbody Fusion in 179 Patients. Asian Spine J 2012;6:89-97.

9. Wang H, Zhang Y, Ma X, Xia X, Lu F, Jiang J. Radiographic Study of Lumbar Sympathetic Trunk in Oblique Lateral Interbody Fusion Surgery. World Neurosurg 2018;116:e380-5.

10. Woods KR, Billys JB, Hynes RA. Technical description of oblique lateral interbody fusion at L1-5 (OLIF25) and at L5-S1 (OLIF51) and evaluation of complication and fusion rates. Spine J 2017;17:545-53.

11. Abe K, Orita S, Mannoji C, Motegi H, Aramomi M, Ishikawa T, et al. Perioperative Complications in 155 Patients Who Underwent Oblique Lateral Interbody Fusion Surgery: Perspectives and Indications From a Retrospective, Multicenter Survey. Spine (Phila Pa 1976) 2017;42:55-62.

12. Ntourantonis D, Tsekouras V, Korovessis P. Delayed Fatal Lumbar Artery Bleeding Following Less Invasive Posterolateral Decompression and Fusion. Spine (Phila Pa 1976) 2018;43:E976-9.

13. Beckman JM, Vincent B, Park MS, Billys JB, Isaacs RE, Pimenta L, Uribe JS. Contralateral psoas hematoma after minimally invasive, lateral retroperitoneal transpsoas lumbar interbody fusion: a multicenter review of 3950 lumbar levels. J Neurosurg Spine 2017;26:50-4.

14. Choy W, Mayer RR, Mummaneni PV, Chou D. Oblique Lumbar Interbody Fusion With Stereotactic Navigation: Technical Note. Global Spine J 2020;10:94S-100S.

15. Takata Y, Sakai T, Tezuka F, Yamashita K, Abe M, Higashino K, Ngamachi A, Sairyo K. Risk Assessment of Lumbar Segmental Artery Injury During Lateral Transpsoas Approach in the Patients With Lumbar Scoliosis. Spine (Phila Pa 1976) 2016;41:880-4.

16. Orita S, Inage K, Sainoh T, Fujimoto K, Sato J, Shiga Y, Kanamoto H, Abe K, Yamauchi K, Aoki Y, Nakamura J, Matsuura Y, Suzuki T, Kubota G, Eguchi Y, Terakado A, Takahashi K, Ohtori S. Lower Lumbar Segmental Arteries Can Intersect Over the Intervertebral Disc in 
the Oblique Lateral Interbody Fusion Approach With a Risk for Arterial Injury: Radiological Analysis of Lumbar Segmental Arteries by Using Magnetic Resonance Imaging. Spine (Phila Pa 1976) 2017;42:135-42.

17. Meeker WQ, Hahn GJ, Escobar LA. Statistical intervals: a guide for practitioners and researchers. Wiley Series in Probability and Statistics. Hoboken, New Jersey: Wiley; 2017.

18. World Medical Association. World Medical Association Declaration of Helsinki: ethical principles for medical research involving human subjects. JAMA 2013;310:2191-4.

19. Nojiri H, Miyagawa K, Banno S, Sakamoto I, Koike M, Sawa M, Iwase Y, Kudo H, Sakai T, Kaneko K. Lumbar artery branches coursing vertically over the intervertebral discs of the lower lumbar spine: an anatomic study. Eur Spine J 2016;25:4195-8.

20. Karunanayake AL, Pathmeswaran A. Anatomical variations of lumbar arteries and their clinical implications: a

Cite this article as: Huang W, Zhou P, Xie L, Huang Z, Zheng C, Ma X, Wang H, Jiang J. The trajectory characteristics and clinical significance of the left-sided lumbar segmental artery: a prospective cross-sectional radio-anatomical study. Quant Imaging Med Surg 2022;12(3):1977-1987. doi: 10.21037/ qims-21-867 cadaveric study. ISRN Anat 2013;2013:154625.

21. Tezuka F, Sakai T, Nishisho T, Takata Y, Higashino K, Takao S, Harada M, Sairyo K. Variations in arterial supply to the lower lumbar spine. Eur Spine J 2016;25:4181-7.

22. Kurunlahti M, Kerttula L, Jauhiainen J, Karppinen J, Tervonen O. Correlation of diffusion in lumbar intervertebral disks with occlusion of lumbar arteries: a study in adult volunteers. Radiology 2001;221:779-86.

23. Li S, Du CZ, Mao SH, Shi BL, Zhu ZZ, Qiu Y. Both structural damage and inflammation of the lumbar spine contribute to the sagittal imbalance in ankylosing spondylitis patients with thoracolumbar kyphosis. Quant Imaging Med Surg 2021;11:362-70.

24. Quillo-Olvera J, Lin GX, Jo HJ, Kim JS. Complications on minimally invasive oblique lumbar interbody fusion at L2-5 levels: a review of the literature and surgical strategies. Ann Transl Med 2018;6:101. 